\title{
Feedback Optimal Control of Low-thrust Orbit Transfer in Central Gravity Field
}

\author{
Ashraf H. Owis ${ }^{\dagger}$ \\ ${ }^{\dagger}$ Department of Astronomy, Space and Meteorology, Faculty of Science, Cairo University \\ ${ }^{\ddagger}$ Department of Aerospace Science and Technology, Politecnico di Milano
}

\section{ABSTRACT}

Low-thrust trajectories with variable radial thrust is studied in this paper. The problem is tackled by solving the HamiltonJacobi-Bellman equation via State Dependent Riccati Equation(STDE) technique devised for nonlinear systems. Instead of solving the two-point boundary value problem in which the classical optimal control is stated, this technique allows us to derive closed-loop solutions. The idea of the work consists in factorizing the original nonlinear dynamical system into a quasi-linear state dependent system of ordinary differential equations. The generating function technique is then applied to this new dynamical system, the feedback optimal control is solved. We circumvent in this way the problem of expanding the vector field and truncating higher-order terms because no remainders are lost in the undertaken approach. This technique can be applied to any planet-to-planet transfer; it has been applied here to the Earth-Mars low-thrust transfer.

\section{INTRODUCTION}

Historically, the optimal low-thrust transfers have been tackled first with indirect and then with direct methods. The former stem from the Pontryagin's maximum principle that uses the calculus of variations [1,2]; the latter aim at solving the problem via a standard nonlinear programming procedure [3]. Even if it can be demonstrated that both approaches lead to the same result [4], the direct and indirect methods have different advantages and drawbacks, but they require in any case the solution of a complex set of equations: the Euler-Lagrange equations (indirect methods) and the Karush-Kuhn-Tucker equations (direct methods). The guidance designed with these methods is obtained in an open-loop context. In other words, the optimal path, even if minimizing the prescribed performance index, is not able to respond to any perturbation that could alter the state of the spacecraft. Furthermore, if the initial conditions are slightly varied (e.g. the launch date changes), the optimal solution needs to be recomputed again. The outcome of the classical problem is in fact a guidance law. expressed as a function of the time, $u=u(t), t \in[t 0, t f]$, being to and tf the initial and final time, and u the control vector, respectively. This paper deals with the optimal feedback control problem applied to the low thrust interplanetary trajectory design. With this approach the solutions that minimize the performance index are also functions of the generic initial state $\mathrm{x} 0$; the outcome is in fact a guidance law written as $u=u(x 0, t 0, t)$, $\mathrm{t} t \in[t 0, t f]$. This represents a closed-loop solution: given the initial conditions $(\mathrm{t} 0, \mathrm{x} 0)$ it is possible to extract the optimal control law that solves the optimal control problem. Moreover, if for any reason the state is perturbed and assumes the new value $\left(t 0^{\prime}, x 0^{\prime}\right)=(x 0+\delta x, t 0+\delta t)$, we are able to compute the new optimal solution by simply evaluating so avoiding the solution of another optimal control problem. This property holds by virtue of the closed loop characteristics of the control law that can be viewed as a one-parameter family of solutions. Due to such property, a trajectory designed in this way has the property to respond to perturbations acting during the transfer that continuously alter the state of the spacecraft. Another important aspect of this approach is the possibility to have robust nominal solutions. Indeed, the optimal feedback control can be analyzed and the control laws being less sensitive to changes in the initial condition can be chosen as nominal solutions. These solutions are said to be robust with respect to the initial conditions. The optimal feedback control for linear systems with quadratic objective functions is addressed through the matrix Riccati equation: this is a matrix differential equation that can be integrated backward in time to yield the initial value of the Lagrange multipliers [2]. The same problem has been tackled in an elegant fashion using the Hamiltonian dynamics and exploiting the properties of the generating functions [5]. With this approach it is possible to devise suitable canonical transformations, satisfying the Hamilton-Jacobi equation, that also verify Hamilton-JacobiBellman equation of the optimal feedback control problem. The generating function technique has been extended to nonlinear dynamical systems supplemented by quadratic objective functions: in this case the vector field is expanded in Taylor series and the optimal control is derived as a polynomial [6]. Nevertheless, the resulting optimal control differs from the one obtained through application of the Pontryagin principle to the nonlinear system since, in the process of series expansion and truncation, the dynamics associated to the high-order terms is neglected. Recently, the nonlinear feedback control of lowthrust orbital transfers has been faced using continuous orbital 
elements feedback and Lyapunov functions [7]. The analytical low-thrust optimal feedback control problem is solved, with modulated inverse-square-distance, in the frame of a nonlinear vector field, the two-body dynamics, supported by a nonlinear objective function by applying a globally diffeomorphic linearizing transformation that rearranges the original problem into a linear system of ordinary differential equations and a quadratic objective function written in a new set of variables [8].

In this work we consider the nonlinear feedback optimal control of the motion of a spacecraft under the influence of the gravitational attraction of a central body, the Sun in our case, and we would like to transfer the spacecraft from Earth to Mars. Both orbits of Earth and Mars around the Sun are assumed to be circular and coplanar. We use both radial and tangential thrust control. The nonlinear dynamics of the system will be factorized in such a way that the new factorized system is accessible. The problem is tackled by solving the State Dependent Riccati Equation (SDRE). The method is applied to Earth-Mars transfer.

\section{Statement of the Problem}

The equations of motion are written in polar coordinates $(r, \theta)$, in the inertial Sun-Centered frame. In order to transfer the spacecraft from Earth to Mars two components of the thrust control are used.The tangential component $T_{\theta}$, and the radial component $T_{r}$.

The equations of motion are:

$$
\ddot{r}-r \dot{\theta}^{2}=T_{r}-\frac{\mu}{r^{2}}, \quad r \ddot{\theta}+2 \dot{r} \dot{\theta}=T_{\theta}
$$

where $\mu$ is the gravitational constant of the $\operatorname{Sun}(1.3271 \times$ $10^{20} \mathrm{~m}^{3} / \mathrm{s}^{2}$ )

So as to use dimensionless variables, we take the unit of distance to be the radius of the circular orbit of the Earth around the Sun, the velocity unit is the velocity of the Earth in its orbit, the frequency is $\omega=\frac{2 \pi}{T}$ where $T=365.25$ days is the periodic time and the unit of time is $\frac{1}{\omega}=58.131343$ days. In this system of units the gravitational constant $\mu$ is unity, and equations (1) are rewritten as:

$$
\ddot{r}-r \dot{\theta}^{2}=T_{r}-\frac{1}{r^{2}}, \quad \ddot{\theta}+2 \frac{\dot{r} \dot{\theta}}{r}=\frac{T_{\theta}}{r}
$$

\section{EQUATIONS OF MOTION IN STATE VARIABLE FORM}

Equations (2) are then written in state variable form. The state vector $\mathrm{x}$ is chosen to be:

$$
\mathbf{x}=\left[\begin{array}{l}
x_{1} \\
x_{2} \\
x_{3} \\
x_{4}
\end{array}\right]=\left[\begin{array}{l}
r \\
\theta \\
\dot{r} \\
\dot{\theta}
\end{array}\right]
$$

and the control vector is :

$$
\mathbf{u}=\left[\begin{array}{l}
u_{1} \\
u_{2}
\end{array}\right]=\left[\begin{array}{l}
T_{r} \\
T_{\theta}
\end{array}\right]
$$

Then Equation (2) can be written in the form :

$$
\dot{\mathbf{x}}=\mathbf{f}(\mathbf{x})+\mathbf{B}(\mathbf{x}) \mathbf{u}
$$

Choosing a suitable factorization equation (5) is rewritten in the factored state variable form :

$$
\dot{\mathbf{x}}=\mathbf{A}(\mathbf{x}) \mathbf{x}+\mathbf{B}(\mathbf{x}) \mathbf{u}
$$

where :

$$
\begin{gathered}
\mathbf{A}(\mathbf{x})=\left[\begin{array}{cccc}
0 & 0 & 1 & 0 \\
0 & 0 & 0 & 1 \\
x_{4}^{2} & -\frac{1}{x_{1}^{2} x_{2}} & 0 & 0 \\
0 & 0 & -\frac{2 x_{4}}{x_{1}} & 0
\end{array}\right] \\
\mathbf{B}(\mathbf{x})=\left[\begin{array}{cc}
0 & 0 \\
0 & 0 \\
1 & 0 \\
0 & \frac{1}{x_{1}}
\end{array}\right]
\end{gathered}
$$

\section{Weak Controllability and Factored CONTROLlability}

Weak Controllability (Accessibility) and Factored Controllability

Weak Controllability (Accessibility) In order for the solution to exist, the system must be weakly controllable (Accessible). A sufficient condition for the system to be weakly controllable on $S \subset \mathbb{R}^{n}$ is that $\forall \mathbf{x} \in S \subset \mathbb{R}^{n}: \operatorname{rank}\left[\Delta_{c}\right]=n$

An algorithm is given below for generating $\Delta_{c}$ :

1) Let $\Delta_{0}=\operatorname{span}(B)=\operatorname{span}\left(b_{i}\right)$

2) Let $\Delta_{1}=\Delta_{0}+\left[a, b_{i}\right]+\left[b_{j}, b_{i}\right]$

3) Let $\Delta_{k}=\Delta_{k-1}+\left[a, d_{j}\right]+\left[b_{i}, d_{j}\right]$

4) Terminate when $\Delta_{k+1}=\Delta_{k}$.

Where $d_{j}$ is the basis of $\Delta_{k-1}$ and the bracket $[f, g]$ is the Lie bracket of $f$ and $g$

$$
[f, g]=\frac{\partial g}{\partial x} f-\frac{\partial f}{\partial x} g
$$

Applying this algorithm to the system (5) to determine its weak controllability we find that:

$$
\Delta_{0}=\left[\begin{array}{cc}
0 & 0 \\
0 & 0 \\
1 & 0 \\
0 & \frac{1}{x_{1}}
\end{array}\right]
$$


whose span is 2 and

$$
\Delta_{1}=\Delta_{2}=\left[\begin{array}{cccc}
0 & 0 & -1 & 0 \\
0 & 0 & 0 & \frac{-1}{x_{1}} \\
1 & 0 & 0 & -2 x_{4} \\
0 & \frac{1}{x_{1}} & \frac{2 x_{4}}{x_{1}} & \frac{x_{3}}{x_{1}^{2}}
\end{array}\right]
$$

with

$$
\begin{gathered}
\operatorname{rank}\left[\Delta_{2}\right]=4=n \\
\forall x_{1} \neq 0
\end{gathered}
$$

In our problem $x_{1} \geq 1$. That is the system is locally controllable.

Factored Controllability For the factored system (6) the controllability is established by verifying that the controllability matrix

$$
\mathbf{M}_{c l}=\left[\begin{array}{llll}
\mathbf{B} & \mathbf{A B} & \mathbf{A}^{2} \mathbf{B} & \mathbf{A}^{3} \mathbf{B}
\end{array}\right]
$$

has a rank equals to $n=4 \forall x$ in the domain. The controllability matrix $\mathbf{M}_{c l}$ for the System (6) is:

$$
\mathbf{M}_{c l}=\left[\begin{array}{cccc}
0 & 0 & 1 & 0 \\
0 & 0 & 0 & \frac{1}{x_{1}} \\
1 & 0 & 0 & 0 \\
0 & \frac{1}{x_{1}} & -\frac{2 x_{4}}{x_{1}} & 0
\end{array}\right]
$$

which has a rank 4.

\section{State Dependent Riccati Equation}

State Dependent Riccati Equation Consider the consider the State Dependent Linear Quadratic Regulator written as follows:

$$
\dot{\mathbf{x}}=\mathbf{A}(\mathbf{x}) \mathbf{x}(t)+\mathbf{B}(\mathbf{x}) \mathbf{u}(t), \quad \mathbf{x}\left(t_{0}\right)=\mathbf{x}_{\mathbf{0}} \in \mathbb{R}^{n}
$$

where $\mathbf{x}(t) \in \mathbb{R}^{n}$ is the state vector and $\mathbf{u}(t) \in \mathbb{R}^{m}$ is the control vector.

The optimization problem is to find the control $\mathbf{u}^{*}$ that minimizes the cost function :

$$
J_{L Q R}=\frac{1}{2} \int_{t_{0}}^{t_{f}}\left(\mathbf{x}^{T} \mathbf{Q} \mathbf{x}+\mathbf{u}^{T} \mathbf{R} \mathbf{u}\right) d t
$$

where $\mathbf{Q}$ and $\mathbf{R}$ are the weight matrices.

State Dependent Riccati Equation The feedback optimal solution of the above problem $\mathbf{u}^{*}$ is given by

$$
\mathbf{u}^{*}(\mathbf{x})=-\mathbf{R}^{-1}(\mathbf{x}) \mathbf{B}^{T}(\mathbf{x}) \mathbf{P}(\mathbf{x}) \mathbf{x}
$$

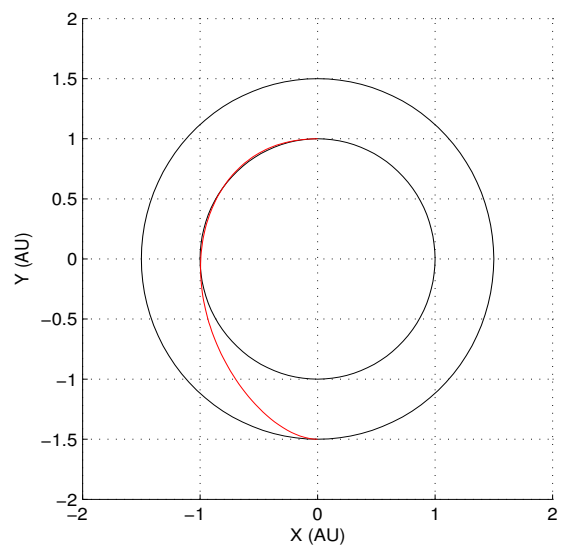

Fig. 1. Trajectory of Earth-Mars transfer in polar coordinates, from $\left[r_{0}=\right.$ $\left.1, \theta_{0}=\pi / 2, \dot{r}_{0}=0, \dot{\theta}_{0}=1\right]$ to $\left[r_{f}=1.5, \theta_{f}=3 \pi / 2, \dot{r}_{f}=0, \dot{\theta}_{f}=\right.$ $0.54433]$

Where $\mathbf{P}(\mathbf{x})$ is obtained by solving the SDRE State Dependent Riccati equation:

$$
\begin{aligned}
\dot{\mathbf{P}}(\mathbf{x})+\mathbf{A}^{T}(\mathbf{x}) \mathbf{P}(\mathbf{x})+\mathbf{P}(\mathbf{x}) \mathbf{A}(\mathbf{x})+\mathbf{Q}(\mathbf{x})- \\
\mathbf{P}(\mathbf{x}) \mathbf{B}(\mathbf{x}) \mathbf{R}^{-1}(\mathbf{x}) \mathbf{B}^{T}(\mathbf{x}) \mathbf{P}^{T}(\mathbf{x})=0
\end{aligned}
$$

We note that the Riccati matrix, $\mathbf{P}(\mathbf{x})$ depends on the choice of $\mathbf{A}(\mathbf{x})$, and since $\mathbf{A}(\mathbf{x})$ is not unique we have multiple optimal solutions.

\section{Optimal ORbit TRANSFER}

Optimal orbit transfer (Numerical examples) In the first example we would like to make an optimal Earth-Mars transfer(i.e. from $(r=1)$ to $(r=1.5))$ in time $t_{f}=4.469$ (295.8 days). The initial angle is $\left(\theta_{0}=\frac{\pi}{2}\right)$ and the final angle is $\left(\theta_{f}=\frac{3 \pi}{2}\right) \cdot \dot{r}_{0}=0$ and $\dot{r}_{f}=0$ for the initial and final orbits. $\dot{\theta}_{0}=\sqrt{\frac{1}{r_{0}^{3}}}=1$ and $\dot{\theta}_{f}=\sqrt{\frac{1}{r_{f}^{3}}}=0.54433105395$. In the second $\theta_{f}=\frac{5 \pi}{2}$ with $t_{f}=6.866$ (397.6 days).

in both examples the matrices $\mathbf{Q}$ and $\mathbf{R}$ are the identity matrices.

$$
\begin{gathered}
\mathbf{Q}=\left[\begin{array}{llll}
1 & 0 & 0 & 0 \\
0 & 1 & 0 & 0 \\
0 & 0 & 1 & 0 \\
0 & 0 & 0 & 1
\end{array}\right] \\
\mathbf{R}=\left[\begin{array}{ll}
1 & 0 \\
0 & 1
\end{array}\right]
\end{gathered}
$$




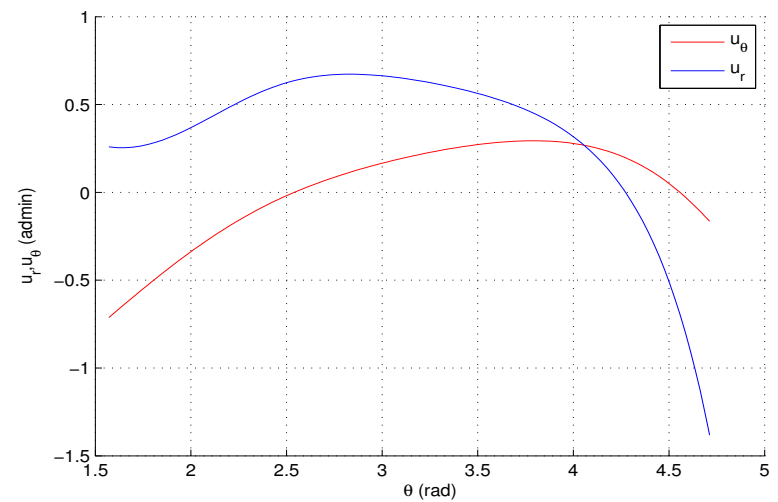

Fig. 2. Controls of the Earth-Mars transfer from $\left[r_{0}=1, \theta_{0}=\pi / 2, \dot{r}_{0}=\right.$ $\left.0, \dot{\theta}_{0}=1\right]$ to $\left[r_{f}=1.5, \theta_{f}=3 \pi / 2, \dot{r}_{f}=0, \dot{\theta}_{f}=0.54433\right]$

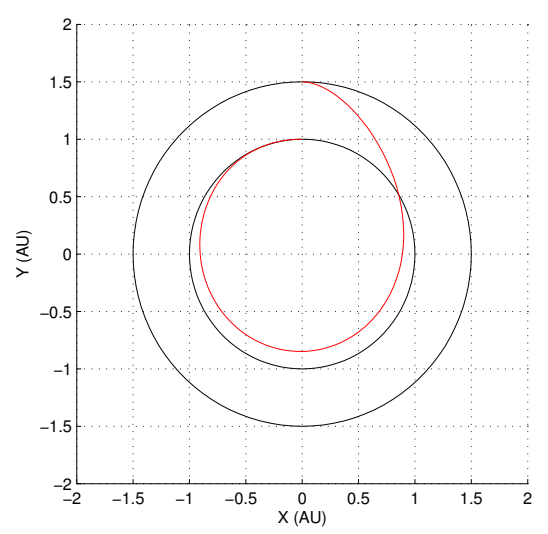

Fig. 3. Trajectory of Earth-Mars transfer in polar coordinates, from $\left[r_{0}=\right.$ $\left.1, \theta_{0}=\pi / 2, \dot{r}_{0}=0, \dot{\theta}_{0}=1\right]$ to $\left[r_{f}=1.5, \theta_{f}=5 \pi / 2, \dot{r}_{f}=0, \dot{\theta}_{f}=\right.$ $0.54433]$

\section{CONCLUSION}

- The nonlinear feedback optimal control can be solved by factorizing the original nonlinear dyanmics into accessible (weakly controllable) linear dynamics of state dependent factors.

- The factrorized problem has been solved using the SDRE

- The method has been implemented to solve feedback optimal control of Earth-Mars orbit tansfer problem.

- The result is valid for any planet to planet transfer.

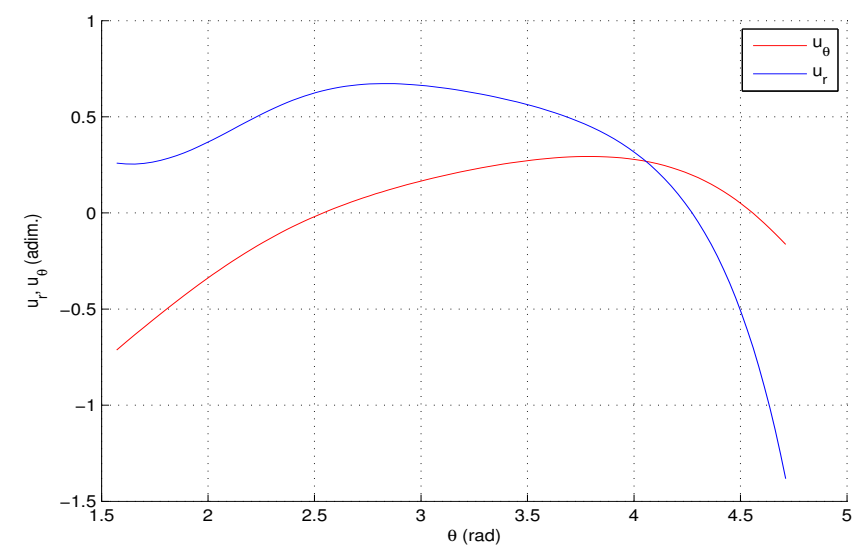

Fig. 4. Controls of the Earth-Mars transfer from $\left[r_{0}=1, \theta_{0}=\pi / 2, \dot{r}_{0}=\right.$ $\left.0, \dot{\theta}_{0}=1\right]$ to $\left[r_{f}=1.5, \theta_{f}=5 \pi / 2, \dot{r}_{f}=0, \dot{\theta}_{f}=0.54433\right]$

\section{ACKNOWLEDGMents}

This project was supported financially by the Science and Technology Development Fund (STDF), Egypt, Grant No 1834 


\section{REFERENCES}

1) L. Pontryagin, V. Boltyanskii, R. Gamkrelidze, and E. Mishchenko, The Mathematical Theory of Optimal Processes, John Wiley \& Sons, New York, 1962.

2) A. Bryson and Y. Ho, Applied Optimal Control. John Wiley \& Sons, New York, 1975.

3) J. Betts, Practical Methods for Optimal Control using Nonlinear Programming, SIAM, 2000.

4) P. Enright and B. Conway, Discrete Approximations to Optimal Trajectories Using Direct Transcription and Nonlinear Programming, Journal of Guidance, Control, and Dynamics, Vol. 15, pp:994-1002, 1992

5) C. Park and D. Scheeres, Solution of Optimal Feedback Control Problems with General Boundary Conditions Using Hamiltonian Dynamics and Generating Functions, Automatica, Vol. 42, pp:869-875, 2006

6) C. Park, V. Guibout, and D. Scheeres, Solving Optimal Continuous Thrust Rendezvous Problems with Generating Functions, Journal of Guidance, Control, and Dynamics, Vol. 29, no. 2, pp:321-331,2006

7) P.Gurfil Nonlinear Feedback Control of Low Thrust Orbital Transfer in a Central Gravitational Field Acta Astronautica, Vol. 60, pp:631-648, 2007

8) F. Topputo, A. Owis, and F. Bernelli-Zazzera Analytical Solution of Optimal Feedback Control for Radially Accelerated Orbits Journal of Guidance, Control, and Dynamics, Vol. 31, No. 5, pp:1352-1359, 2008

9) K. D. Hammetty,C. D. Hallz, and D. B. Ridgelyx,Controllability Issues in Nonlinear StateDependent Riccati Equation Control, Journal of Guidance, Control, and Dynamics vol.21 no.5, PP:767-773, 1998 